\title{
Aetiolog I cal Pattern of Premature Ventricular and Atrial Complexes and their Consequence on Morbidity and Mortality
}

\author{
AKM HUMAYON KABI R, ${ }^{1}$ MOHAMMAD RAFI QUL ISLAM, ${ }^{1}$ PRODI P KUMAR BI SWAS, ${ }^{1}$ MUHAMMED SYEDUL \\ ALAM, ${ }^{2}$ SHEKHAR KUMAR MONDAL, ${ }^{3}$ MD. ENAMUL KARI M, ${ }^{4}$ MA AZHAR ${ }^{5}$
}

\begin{abstract}
Background: Arrhythmias are often a manifestation of structural heart diseases but may occur in the context of non cardiac diseases even in an otherwise normal heart. It may be supraventricular or ventricular. Among the arrhythmias premature complexes (ventricular \& atrial) are most common type of arrhythmia occurs in patients with or without significant heart disease.

Objective: In this study, the aim was to find out the aetiological pattern of premature ventricular and atrial complexes and their consequence on morbidity and mortality, demographical pattern of patients with ectopic beats, to find out aetiological factors leading to development of premature complexes, to find out common clinical presentations and to differentiate the PVCs which are more benign

Methods: It was a cross sectional study. This study was carried out in the Department of Medicine and Department of Cardiology of Sir Salimullah Medical College and Mitford Hospital, Dhaka from I ${ }^{\text {st }}$ May 2008 to $15^{\text {th }}$ September 2008. A total 50 consecutive patients irrespective of age, sex and occupation having atrial and ventricular premature complexes on ECG attending at OPD and admitted in indoor were included in this study.

Results: Among the three types of premature complexes 9 patients with APCs (also includes 2 patients of premature junctional complex) and 4 I patients with PVCs were included. The ratio of patients with PVCs and APCs in this study.8.2: I.8. In this study 4 I patients of PVCs were studied. Among them 37 patient that is $90.3 \%$ patients had structural heart disease and the commonest cause was IHD (63.4\%), 9.7\% had HTN with LVH, 7.3\% had DCM, 4.87\% had HOCM, one patient had myocarditis ( $2.4 \%$ ) and one had MVP with sick sinus syndrome (2.4\%). Among the 9 patients of APC 4 patients that is $44.44 \%$ were suffering from COPD with corpulmonale which was the most commonest cause of APCs.

Conclusions: Ventricular arrhythmias are more common than atrial arrhythmia and IHD is the single most common cause of arrhythmia. Ectopic beats in patients with otherwise normal hearts are more prominent at rest and disappear with exercise. Treatment is not necessary, unless the patient is highly symptomatic.
\end{abstract}

Key words: Atrial premature complexes (APCs), Premature ventricular complexes (PVCs).

\section{Introduction}

The heart starts beating from $4^{\text {th }}$ week of intrauterine life in a rhythmic way which is unique in nature. There is perhaps no other example of such perfect rhythmicity as that of heart beat. Any deviation from normal rhythm of heart is known as cardiac arrhythmia. Arrhythmias are often a manifestation of structural heart diseases but may occur in the context of non cardiac diseases even in an otherwise normal heart. ${ }^{1,2}$

1. Assistant Professor of Medicine, Dhaka Medical College

2. Assistant Professor of Medicine, Chittagong Medical College

3. Assistant Registrar of Medicine, Dhaka Medical College Hospital

4. Professor and Head, Department of Medicine, Dhaka Medical College

5. Professor and Head, Department of Medicine, SSMC and Mitford Hospital

Correspondence: Dr. AKM Humayon Kabir, Assistant Professor of Medicine, Dhaka Medical College. Room no- 531, DMCH-2. Mobile: 01711137528, Email: drakmhkabir@gmail.com
Arrhythmias may be supraventricular or ventricular. Among the arrhythmias premature complexes (ventricular \& atrial) are most common type of arrhythmia occurs in patients with or without significant heart disease. ${ }^{3}$

Premature atrial complexes (APCs) are found in about $60 \%$ of normal adult on holter monitoring and which are usually asymptomatic and benign and no treatment is required. Sometimes when it is very frequent may produce symptoms like palpitation and may herald the onset of paroxysmal SVT or atrial fibrillation. ${ }^{4}$ Then treatment may be useful.

Premature ventricular complexes (PVCs) are very infrequent in 12 leads ECG in asymptomatic individuals. The Framingham heart study (with one hour ambulatory ECG) shows that the prevalence of one or more PVCs was 33\% in men and 32\% women without CAD (Coronary Artery Disease). Among the CAD the prevalence was $58 \%$ in male and $49 \%$ in female. ${ }^{5,6}$ 
PVCs are very common in CAD (up to $80 \%$ after MI) and also common in hypertension, LVH (Left Ventricular Hypertrophy), cardiomyopathy. MVP (mitral valve prolapse) and some drugs ie, digoxin and in extreme bradycardia as escape beat. ${ }^{7}$

Although PVCs are very common in healthy adult, sometimes specially if it is associated with structural heart disease it may lead to ventricular tachycardia (VT) and which may degenerate to ventricular fibrillation and which is one of the most common mechanism of sudden death. ${ }^{8,9}$

So, presence of PVCs and other ventricular arrhythmia often engender an anxiety to the patient and also to the physician and may lead to unnecessary investigation and inappropriate treatment (either over treated or under treated). So one must therefore attempt to distinguish those PVCs are benign requiring only reassurance and follow up from those which are potentially more sinister and require aggressive intervention., ${ }^{2,9}$

Again, treatment paradigm used in 1970s. 1980s was to eliminate PVCs after Myocardial infarction (MI), has now been changed. As domination of PVCs with available anti arryhthmic drugs (class 1), may increase risk of sudden death with its pro arryhthmic effect $(\mathrm{CAST})^{10}$. A study on ectopic beats will therefore reveal the nature, severity and their outcome.

\section{Methods}

It was a cross sectional study. This study was carried out in the Department of Medicine and Department of Cardiology of Sir Salimullah Medical College and Mitford hospital, Dhaka from $1^{\text {st }}$ May 2008 to $15^{\text {th }}$ September 2008. A total 50 consecutive patients irrespective of age, sex and occupation having atrial/ ventricular premature complexes on ECG attending at OPD and admitted in indoor were included in this study.

The inclusion criteria for selection of the study population were i) age above 20 years. ii )Random selection of the patient having PVCs or APCs on ECG. iii) Patients having complaints of palpitation or on examination revealed dropped beat or irregular pulse followed by ECG showing PVCs or APCs are included in this study. Exclusion criteria were as follows- i) Patient dropped out before investigation were completed or died before complete diagnosis. ii) Patient who did not give consent.

There are three types of premature complexes i)Atrial premature complexes. (APCs) ii) AV nodal premature complexes. iii) Ventricular premature complexes. (PVCs). After establishing the diagnosis by a 12 lead ECG, every case was studied in detail, including a proper history taking ,complete meticulous physical examinations and relevant investigations were done. After a complete history taking, a detailed physical examinations was done with special emphasis on Pulse in details, BP, JVP and examination of precordium.

After the diagnosis was made, following investigations were done routinely These were Blood for (1) TC, DC, ESR, Hb\%, (2) Blood urea and serum creatinine, (3) Blood and urine sugar, (4) Serum cholesterol, (5) ECG, (6) Chest x-ray and (7) Echocardiography. Following investigations were also done for selective cases as indicated. (1) Serum troponin l, CK.-MB (2) T3, T4 and TSH (3) Holtcr monitoring, (4) ETT (5) S. electrolytes (6) CAG, etc.

After confirmation of diagnosis (Type of ectopics and aetiology) appropriate treatment was given and the patients were periodically follow up (on discharge those ectopic were subsided and after 1 month those ectopics not subsided (with "ECG”) to see the outcome.

\section{Results}

A total of 50 patients were included in the study. Among the three types of premature complexes 9 patients with APCs (also includes 2 patient of premature junctional complex) and 41 patient with PVCs were included. The ratio of patients with PVCs and APCs in this study 8.2: 1.8 (Table -1).This study, age range was from 20 years to 75 years. The maximum age incidence of PVCs with IHD group was 51 to 60 years which was $42.33 \%$ of this group. Next common group was 61 to 70 years (34.6\%). In non IHD group the Maximum age incidence was 21 to 30 years (40\%) Next one was 31 to 40 years (Table-II). In this study 32 patients were male and 18 patients were female.

Table-I

Types of premature complexes (APCs/PVCs).

\begin{tabular}{lcc}
\hline $\begin{array}{l}\text { Types of premature } \\
\text { complexes }\end{array}$ & $\begin{array}{c}\text { Number of } \\
\text { patients }\end{array}$ & $\begin{array}{c}\text { Percentage } \\
(\%)\end{array}$ \\
\hline (1) PVCs & 41 & $82 \%$ \\
(2) APCs & 9 & $18 \%$ \\
Total number of patients & 50 & \\
\hline
\end{tabular}

Overall male: female ratio was 6.4 : 3.6. In PVCs with IHD Group male: female ratio was 6.9 : 3.1. PVCs with non IHD Group male and female ratio was 5.3 : 4.7. In APCs Group male and female ratio was 6.6 : 3.3 ( Table-III). In this study PVCs in IHD group all (that is 100\%) patient had present or past history of chest pain. 20 of 26 patients gave history of 
palpitation which was $76.9 \%$. 38.46\% with breathlessness, $30.76 \%$ with cough, $15.38 \%$ patients were presented with syncopal attack. Among the non IHD group present with palpitation, 12 of 15 patient that is $80 \%$ followed by cough and chest pain (non specific). Among the APCs group 100\% patient was present with cough, 89\% with breathlessness and only $22 \%$ gave history of palpitation. (Table-IV). In this study 41 patients of PVCs was studied. Among them 37 patients that is $90.3 \%$ patient had structural heart disease and the commonest cause was IHD (63.4\%), 9.7\% had HTN with LVH, 7.3\% had DCM, 4.87\% had HOCM, one patient had myocarditis 2.4\%) and one had MVP with sick sinus syndrome (2.4\%). 9.7\% patients that is 4 out of 41 had no structural heart disease, among them one was suffering anxiety disorder in other 3 patients no cause had been detected, although all were either smoker or had history of taking tea or coffee (Table-V).

In this series 9 patients of APCs was studied. Among them 4 patient that is $44.44 \%$ were suffering from COPD with corpulmonale which was the most common cause of APCs. All the 4 patients were also getting salbutamol and theophylline which may precipitate the development of APCs. 2 patients had MVD and 2 patients had ASD (Table-VI).

Table - II

Incidence of different Age group.

\begin{tabular}{lcccccc}
\hline Age & No. of Patient & Percentage (\%) & No. of Patient & Percentage(\%) & No. of Patient & Percentage(\%) \\
\hline$<20$ years & 0 & & 0 & & 0 & \\
$21-30$ & 0 & & 6 & $40 \%$ & 2 & $22.22 \%$ \\
$31-40$ & 1 & $3.8 \%$ & 5 & $33.33 \%$ & 2 & $22.22 \%$ \\
$41-50$ & 3 & $1 i .53 \%$ & 2 & $13.33 \%$ & 1 & $11.11 \%$ \\
$51-60$ & 'll & $41.33 \%$ & 2 & $13-33 \%$ & 1 & $11.11 \%$ \\
$61-70$ & 9 & $34.6 \%$ & 0 & & 2 & $22.22 \%$ \\
$71-80$ & 2 & $7.6 \%$ & 0 & & 1 & $11.11 \%$ \\
\hline Total & 26 & $100 \%$ & 15 & $100 \%$ & 9 & $100 \%$ \\
\hline
\end{tabular}

Table - III

Incidence of different sex groups.

\begin{tabular}{|c|c|c|c|c|c|c|}
\hline \multirow[t]{2}{*}{$\overline{\text { Sex group }}$} & \multicolumn{2}{|c|}{ PVCs in IHD group } & \multicolumn{2}{|c|}{ PVCs in non IHD group } & \multicolumn{2}{|c|}{ APCs } \\
\hline & No.of Patient & Percentage(\%) & No. of j Patient & Percentage(\%) & No. of Patient & Percentage(\%) \\
\hline$\overline{\text { Male }}$ & 18 & $69 \%$ & 8 & $53 \%$ & 6 & $66.6 \%$ \\
\hline Female & 8 & $31 \%$ & 7 & $47 \%$ & 3 & $33.33 \%$ \\
\hline Total & 26 & $100 \%$ & 15 & $100 \%$ & 9 & $100 \%$ \\
\hline
\end{tabular}

Table - IV

Distribution of symptoms.

\begin{tabular}{|c|c|c|c|c|c|c|}
\hline \multirow{2}{*}{$\begin{array}{l}\text { Symptoms } \\
\text { (Cardiovuscular) }\end{array}$} & \multicolumn{2}{|c|}{ PVCs in IHD group } & \multicolumn{2}{|c|}{ PVCs in non IHD group } & \multicolumn{2}{|c|}{ APCs } \\
\hline & No. of Patient & Percentage & No. of Patient & Percentage(\%) & No. of Patient & Percentage (\%) \\
\hline Palpitation & 20 & $76.92 \%$ & 12 & $80 \%$ & 2 & $22.22 \%$ \\
\hline Chest Pain & 26 & $100 \%$ & 6 & $40 \%$ & 3 & $33.33 \%$ \\
\hline Breathlessness & to & $38.5 \%$ & 5 & $33.33 \%$ & 8 & $88.88 \%$ \\
\hline Cough & 8 & $30.76 \%$ & 7 & $46.66 \%$ & 9 & $100 \%$ \\
\hline SyncopalAsttack & 4 & $15.38 \%$ & 2 & $13.33 \%$ & & 1 \\
\hline Leg swelling & 3 & $11.53 \%$ & 2 & $13.33 \%$ & 2 & $22.22 \%$ \\
\hline Feature of non & 4 & $15.38 \%$ & & & 5 & $55.55 \%$ \\
\hline \multicolumn{7}{|l|}{ Cardiovuscular } \\
\hline Symptoms Others & & & & & & \\
\hline
\end{tabular}


Table - V

Aetiological pattern of PVCs in structural heart disease group.

\begin{tabular}{lcc}
\hline Aetiology & No. of Patient & Percentage (\%) \\
\hline PVCs with II ID & 26 & $100 \%$ \\
ACS (STEMI, NSTEMI, UA) & 20 & $76.92 \%$ \\
OMI/Ischemia & 6 & $23.08 \%$ \\
PVCs with non IHD & 15 & $100 \%$ \\
HTN & 4 & $26 \%$ \\
DCM & 3 & $20 \%$ \\
HOCM & 2 & $13 \%$ \\
Others (MVP, Myocarditis) & 2 & $13 \%$ \\
\hline
\end{tabular}

Table-VI

Aetiological pattern of APCs.

\begin{tabular}{lcc}
\hline Aetiology & No. of Patient & Percentage (\%) \\
\hline Mitral Vulvular & 2 & $22.22 \%$ \\
heart disease & & \\
ASD & 2 & $22.22 \%$ \\
COPD with Corpulmonale & 4 & $44.44 \%$ \\
RTI & 1 & $11.11 \%$ \\
\hline Total No. Patient & 9 & $100 \%$ \\
\hline
\end{tabular}

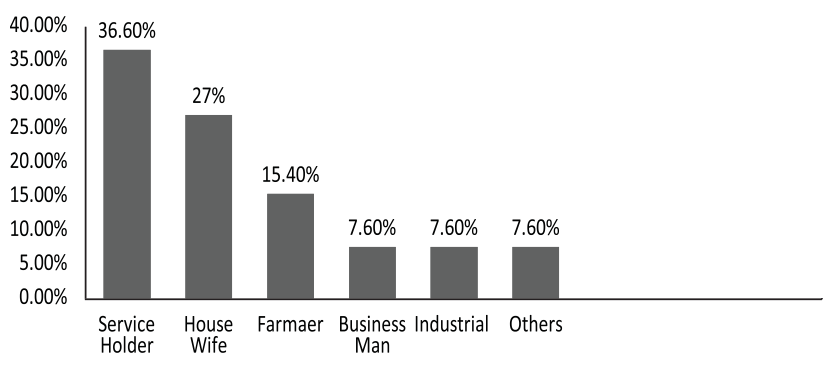

Fig-I: Shows Occupation Incidence of PVC s in IHD group

\section{Discussion}

PVCs arc very common in coronary artery disease (CAD) specially after MI. Among the PVCs in IHD group in this study, the maximum age incidence was 51-60 years which correlates with other observation. This is the vulnerable age group for MI with complications. Followed by 61-70 years. Among the PVCs with non IHD group, the maximum age incidence was 21 to 30 years followed by 31 to 40 years age group, as HOCM, MVP, DCM are commonly present in this age group an these disease were found responsible for ectopics. ${ }^{11,12}$
In this study, PVCs with IHD group male and female ratio was $6.9: 3.1$ Since IHD is common in male, which correlates with Framingham heart study. Among the non IHD group the male and female ratio of incidence of PVCs was 5.3 : 4.7 indicating no significant difference However, among the APCs group male and female ratio was 6.2 : 3.8 indicating it is more common in male population.

In this study IHD related PVCs and other arrhythmias commonly occurred in sedentary workers (includes service holder, $34.5 \%$, and the female house wife $27 \%$ total $61.5 \%$ which was almost same as subash et al ${ }^{13}$, where the incidence of this type of arrhythmias among the sedentary workers was $45.7 \%$ but where teachers $8 \%$ and house wife $10 \%$ was not included in sedentary group. The next maximum occupational group was farmer and worker related to farm which was $15.4 \%$ who are the largest occupational group in our country. Non IHD related PVCs and APCs was also most common in sedentary workers group, this clearly indicates that physical activity or exertion reduces the incidence of ectopics.

In this series, PVCs in IHD group 61.5\% patients were smoker as whole. But among the male $83.3 \%$ patients were smoker which correlates with other studies, Subash et al ${ }^{13}$ (81.8\%), Jalal Uddin et al ${ }^{14}$ (82\%), Aberge et al ${ }^{20}$ (78\%) and. In one study of cardiac arrhythmias suppression trail $(\mathrm{CAST})^{10}$ it was found that smoking increase APCs overall of the patient with IHD. Among the APCs group; 55.5\% patient were smoker.

In this study PVCs in IHD group 100\% patient give present or past history of ischemic type chest pain. Chest pain was also presenting complaints in $92.6 \%$ and $96 \%$ of IHD patient in other series, more or less consistent with this study. 76.9\% patients in this study presented with palpitation, $38.46 \%$ with occasional breathlessness, $30.76 \%$ with cough, $15.38 \%$ with syncopal attack, and $11.54 \%$ with leg edema, all are being common cardiac symptoms. Among the non IHD group the most common presentation was palpitation (80\%). followed by cough (46.6\%). Among the APCs group 100\% patients had cough and $89 \%$ had breathlessness, which was next common presentation.

IHD risk factors among the IHD with PVCs group: 83\% male patients were smoker. 33.8\% had hypertension, 23\% were diabetic and $19.6 \%$ patient had dyslipidemia, $11.53 \%$ patient had family history of premature CAD which was almost similar to other study.

Among the non IHD group, one of two patients with HOCM had history of sudden death of his elderly brother at the age of 30 . 
Among the APCs group 55.5\% was smoker, all the 4 COPD patient was getting sulbutamol, tablet theophylline which might have precipitated the development of APCs. ${ }^{15,16}$ Most of them also gave history of taking tea, coffee or betal nut which also may be the precipitant.

Among the IHD group with PVCs most of them was suffering from acute coronary syndrome (ACS) with OMI with or without heart failure. Among them 76.92\% (20 of 26) patients was suffering acute coronary syndrome. 46\% (12 of 26) patients was present with STEMl (ST elevation myocardial infarction), 15\% with NSTEMI (non ST elevation myocardial infarction), 15\% with UA (unstable angina). 23\% (6 of 26) patients present with OMI (old myocardial infarction). Among the patients with no structural Heart diseases, PVCs was also common. 26.6\% (2 in 15) patients suffering from no heart disease present with PVCs which correlates with Framing Ham study PVCs also common in non IHD structural Heart diseases, among them 26\% patient had HTN, 20\% had DCM, 13\% had HOCM and others was 13\% (MVP with sick sinus syndrome, myocarditis).

Among the APCs group most common cause was COPD with or without corpulmonale. $44.45 \%$ (4 of 90) followed by mitral valvular disease (22.2\%). 22.2\% patient had ASD (atrial septal defect). $11.1 \%$ patient had respiratory tract infection (RTl). Most of the symptomatic patient with PVCs or APCs was treated with anti arrhythmic drugs like betablocker or digoxin or amiodarone or and anti ischemic drugs or other anti failure drugs. Most of the asymptomatic patient did not take any treatment except explanation, and reassurance. ${ }^{17,18,19}$

Out come: Among the study group, 23\% patient had developed Acute LVF at presentation or during hospitalized period. They were treated appropriately but one patient was expired.

7.7\% patients developed 2:1 block, requiring TPM. (Temporary pace-maker) with complete recovery, 3.8\% patient developed complete Heart block (CHB) requiring TPM followed by PPM (permanent pace maker), 7.7\% patient developed LBBB without further deterioration.

15.4\% patients developed VT (ventricular tachycardia) at presentation or during hospitalized period was treated with either iv lignocaine or amiodarone, followed by oral amiodarone or beta-blocker but one patient degenerate to ventricular fibrillation (VF), followed by death, could not rescue even after defibrillation and other appropriate measures.

Among the Non IHD group, 6.7\% patient with myocarditis developed cardiogenic shock requiring ionotropic support with full recovery.
6.7\% patient with HOCM developed 2 episodes of syncopal attack, treated with IV lignocaine followed by oral amiodarone and beta-blocker. The another patient with HOCM with $6^{\text {th }}$ months pregnancy with CCF was ultimately died even after appropriate treatment.

\section{Death:}

7.31\%. Patient with PVCs group had died (3 out of 41 patients with PVCs). 2.5\% (One) of them developed acute LVF with cardiogenic shock following acute extensive anterior MI. $16.6 \%$ patient out of six patients with acute LVF died correlates with Subash et al ${ }^{13}$ and Farid et al. ${ }^{19}$ One patient developed VT (ventricular tachycardia which degenerate to VF and ultimately expired. One of four patients with VT that is $25 \%$ VT patient was died. One patient with HOCM died.

\section{Conclusion}

Arrhythmia is a common cardiac problem. This study shows ventricular arrhythmias are more common than atrial arrhythmia. And IHD is the single most common cause of arrhythmia. The commonest mode of presentation of arrhythmia is palpitation along with feature of etiology commonly chest pain. ECG is the most useful test for diagnosis of arrhythmia. Treatment of cause is the primary need. Some patients require, only explanation and reassurance and others require antiarrhythmic drugs or defibrillation. Some types of arrhythmia (Premature complexes) may degenerate to life threatening tachyarrhythmia or fibrillation which eventually lead to death if untreated. Therefore physician should have sound knowledge of ECG so that they can diagnose early, manage promptly and refer early to a near bycentre having all facilities to deal with arrhythmia.

\section{Conflict of Interest: None}

\section{References}

1. Josepi Ison ML, Zimetbaum P. The trachyarrhythmias, The bradyarrhythmias, Harrison's Principles of Internal Medicine $16^{\text {th }}$ ed. $2005 ; 1334-58$.

2. Bikkina M. Larson MG, Levy D: Prognostic implications of asymptomatic ventricular arrhythmias: the Framingham Heart Study. Ann Intern Med 1992 Dec 15;117(12):990-6.

3. The Cardiac Arrhythmia Suppression Trial II Investigators:Effect of the antiarrhythmie agent moricizine on survival after myocardial infarction. N Engl J Med 1992 Jul 23:327 (4):227-33.

4. Desmond G. Julian, Disorder of rate, Rhythm, and conductions, cardiology $7^{\text {th }}$ ed.:177-222.

5. Rhythmical excitation of heart Guyton \& Hall, Text book of Medical physiology, Saunders, 2001, 10 ${ }^{\text {th }}$ ed.:107-13. 
6. Mason JW: A comparison of electrophysiologic testing with Holler monitoring to predict versus Electrocardiogtraphic Monitoring Investigators. N Engl J Med. 1993;329(7): 445-51.

7. KennedyFIl, Whitlock JA, Sprague MK, et al:Long-term follow-up of asymptomatic healthy subjects with frequent and complex ventricular ectopy. $\mathrm{N}$ Engl J Med. 1985;312(4):193-7.

8. Brad Ford C. Lipman A.B. Grading systems for ventricular ectopy, ECG Pocket guide, $1^{\text {st }}$, ed. 1990, Jaypee, Book:6373,142 .

9. Alpest MA, Mukcrji V, Btkkina M, et al: Pathogenesis, recognition, and management of common cardiac arrhythmias. Part I: Ventricular premature beats and tachyarrhythmias. Sout Med J 1995;88(1):1-. 21.

10. Epstein AEm I lallstrom AP, WJ, et al: Mortality following ventricular arrhythmia suppression by encainide, flecainide, and moricizine after myocardial infarction. The original design concept of the Cardiac Arrhythmia Suppression Trial (CAST). JAMA 1993;24(20):2451-5.

11. Aberg A, Laurice DM, Friedric 1. Cessation of smoking after myocardial infarction Br Heart J. 1983;49:416-22.
12. Barman, RabindraNath, Patterns of arrhythmia after acute myocardial infarction during hospital period. Dissertation, FCPS (Medicine), Part- 1l. BCPS, 2005.

13. Subash D, Zaher A, Hossain M, Morshed M, Nabi MN. Clinical profile of acute $\mathrm{Ml}$ Cases admitted in CCU of CMC11. Bangladesh Heart Journal 1992;7:45-52.

14. Jalaluddin A, Malik A. A study of relation of smoking with myocardial infraction in 100 cases Bangladesh Heart Journal 1986;(1):23-25.

15. Rahman MA. Clinical pattern of arrhythmias. Dissertation, FCPS (Medicine), Part-ll. BCPS, 1978

16. Andersson S, Osterlind PO, Holmboe G, Windblad B: Twenty-four- hour electrocardiograph in a healthy elderly population. Gerontology 1988;34(3):139-44.

17. Kahhar MA. Patterns of cardiac arrhythmias in mitral valvular disease.

18. Henry WL et al. Relation betv/eenjechocardiographically determined left atrial size and atrial fibrillation. Circulation 1976;53:273.

19. Farid N, Bari S. Experience of treatment of myocardial infarction at Mymensingh Medical College 1993;(2):26-30.

20. Aberg A, Laurice DM, Friedric 1. Cessation of smoking after myocardial infarction Br Heart J 1983;49:416-22. 